\title{
Error Analysis of Miller's Recurrence Algorithm
}

\author{
By F. W. J. Olver
}

Abstract. Miller's algorithm is a device for computing the most rapidly decreasing solution of a second-order linear difference equation. In this paper strict upper bounds are given for the errors in the values yielded by the algorithm, and general conclusions are drawn concerning the accuracy of the process.

1. Introduction. The recurrence algorithm we are concerned with here was originally devised by J. C. P. Miller for the computation of tables of the modified Bessel function $I_{r}(x)$ ([1], page xvii). The process was as follows. "Dummy" or "trial" values 1 and 0 are assigned to $I_{n}(x)$ and $I_{n+1}(x)$, respectively, where $n$ is an arbitrarily chosen large positive integer. The recurrence relation

$$
I_{r-1}(x)=(2 r / x) I_{r}(x)+I_{r+1}(x)
$$

is then applied for $r=n, n-1, \cdots, 1$ in turn to generate a corresponding sequence of trial values of $I_{r}(x)$. In consequence of the initial conditions the $r$ th trial value is equal to

$$
\lambda\left\{I_{r}(x)+(-)^{n-r} \frac{I_{n+1}(x)}{K_{n+1}(x)} K_{r}(x)\right\}
$$

where $\lambda$ is independent of $r$ (in fact $\lambda=x K_{n+1}(x)$, but this expression is not used computationally). Now as $n \rightarrow \infty, I_{n+1}(x)$ decreases rapidly and $K_{n+1}(x)$ increases rapidly. Hence by taking $n$ to be sufficiently large we can ensure that for any given value of $r$, indeed for any given bounded range of values of $r$, the second term in the braces in (1.02) is negligible, so that the trial values effectively reduce to $\lambda I_{r}(x)$. The "normalizing factor" $\lambda$ can be determined, for example, by dividing the trial value of $I_{0}(x)$ by the actual value of this function obtained from tables. Alternatively, the normalization can be effected by application of either of the relations

$$
\begin{aligned}
& I_{0}(x)-2 I_{2}(x)+2 I_{4}(x)-\cdots=1, \\
& I_{0}(x)+2 I_{1}(x)+2 I_{2}(x)+\cdots=e^{x} .
\end{aligned}
$$

The use of tables of Bessel functions is then avoided altogether, a striking feature which is of especial importance in automatic computation.

Following Miller's original use, similar processes have been used or described for other computations of Bessel functions ([2]-[9]), and also for the computation of repeated integrals of the error function ([10], [11]) and associated Legendre functions [12]. In some of these references, beginning with [2], the process is used in a form involving the ratios of the successive wanted functions; for this reason it is sometimes called the Ratio Method [8].

Recently, a method for solving ordinary differential equations in series of Chebyshev polynomials has been developed by C. W. Clenshaw [13] which has

Received May 13, 1963. 
features in common with the Miller algorithm. Indeed, for the differential system

$$
y^{\prime \prime}(x)+k^{2} y(x)=0, \quad y(0)=\frac{1}{2}, \quad y^{\prime}(0)=0,
$$

Clenshaw's method of solution applied to the range $-1 \leqq x \leqq 1$ becomes exactly the algorithm for the calculation of the Bessel functions $J_{r}(k)$, with a normalizing condition of the form (1.03).

For the general homogeneous linear difference equation of the second order

$$
f_{r-1}+p_{r} f_{r}+q_{r} f_{r+1}=0,
$$

together with a normalizing relation of the form

$$
m_{0} f_{0}+m_{1} f_{1}+m_{2} f_{2}+\cdots=1,
$$

in which the $m_{r}$ are given numbers, the Miller algorithm may be formalized as follows. We construct a sequence of trial values $a_{r}$ according to the equations

$$
a_{n+1}=0, \quad a_{n}=1, \quad a_{r-1}=-p_{r} a_{r}-q_{r} a_{r+1} \quad(r=n, n-1, \cdots, 1),
$$

and thence a further sequence

$$
\varphi_{r}=a_{r} / \lambda
$$

in which $\lambda$ is determined by

$$
\lambda=m_{0} a_{0}+m_{1} a_{1}+\cdots+m_{n} a_{n} .
$$

In the case $m_{1}=m_{2}=\cdots=0$, that is, when $f_{0}$ is prescribed, W. Gautschi [11] has shown, by an extension of the argument given in [1], that if (1.06) possesses a second solution $g_{r}$ which is non-vanishing and such that

$$
f_{r} / g_{r} \rightarrow 0 \text { as } r \rightarrow \infty
$$

then for any fixed $r$

$$
\varphi_{r} \rightarrow f_{r} \text { as } n \rightarrow \infty .
$$

Gautschi has also shown, in unpublished work, that with suitable conditions the proof can be further extended to include the general normalizing condition (1.07).

These results of Miller and Gautschi provide a satisfactory account of the convergence of the algorithm. They show that with appropriate conditions each $f_{r}$ can be computed to any desired accuracy by taking $n$, the point at which the backwards recurrence begins, to be large enough, $n \geqq n_{r}$, say. These results do not, however, supply a value for $n_{r}$ in any given example. Sometimes a suitable value for $n_{r}$ can be determined by use of known analytical properties of the solutions of the difference equation, as in [4], [8] and [11]. An alternative procedure is to compare the results of applying the algorithm for different values of $n$, described, for example, in [5] and [9]; this is generally an adequate check on the convergence in practice, though not entirely satisfactory from a mathematical standpoint.

In the present paper, we do not use the condition (1.11). Instead, we make other assumptions concerning the nature of the solutions of the difference equation, from which we determine, by quite elementary analysis, simple strict error bounds for the results yielded by the algorithm. From these bounds we are able to draw interesting conclusions concerning the general accuracy of the method. 
We shall suppose at first that the algorithm is applied with the use of exact arithmetic. Later, in $\S 4$, we allow for the fact that in practice the trial values $a_{r}$ are generally rounded at each step of the recurrence, thus introducing another possible source of error. Only in [8] does the effect of rounding the $a_{r}$ appear to have been analysed before, but the treatment in this reference is of an approximate character, and is also less general than that presented below.

2. Assumptions. The basic assumption we make in this paper is that the difference equation (1.06) has independent solutions $f_{r}$ and $g_{r}$ such that $\left|f_{r}\right|$ has acquired a moderately rapidly decreasing character and $\left|g_{r}\right|$ a moderately rapidly increasing character at the stage $(r=n)$ at which the algorithm is applied. More precisely, we suppose that $f_{n}$ and $g_{n+1}$ do not vanish, that

$$
\rho=\left|f_{n+1} / f_{n}\right|
$$

is small, and that the least quantity $\sigma$ such that the inequalities

$$
\left|g_{r} / g_{n+1}\right| \leqq \sigma^{n+1-r}
$$

hold for $r=0,1, \cdots, n$, also is small. Here $n$ is an arbitrary positive integer, and $\rho, \sigma$ in general depend on $n$, but it is unnecessary to indicate this explicitly in the notation.

We further suppose that the application of the algorithm is delayed until the series on the left of (1.07) is converging reasonably rapidly after its $(n+1)$ th term. To express this precisely, we introduce the notation

$$
m=\max _{0 \leqq r \leqq n}\left|m_{r}\right|, \quad \tau=\left|\frac{1}{m f_{n}} \sum_{r=n+1}^{\infty} m_{r} f_{r}\right| .
$$

Then the assumption of this paragraph is that $\tau$ is small. We may notice that this condition is automatically fulfilled by the simplest type of normalization, in which $f_{0}$ is prescribed (and non-zero), for in this case $\tau$ vanishes.

Perhaps it should be stressed that actual values for the quantities $\rho, \sigma$ and $\tau$ do not need to be known in any given example, a mere knowledge that they are small suffices for the application of the error analysis of the following sections.

In the case of Miller's Bessel-function example, given in $\$ 1$, the above conditions are fulfilled provided that $n$ is taken to be somewhat larger than the argument $x$. This can be verified by inspecting tables of Bessel functions, or alternatively by examining the asymptotic behaviour of the Bessel functions for large orders. Similar verifications might be made in the case of other well-known special functions, for example, Legendre functions.

In any case, however, it is frequently quite straightforward to determine the main features of the asymptotic behaviour of $f_{r}$ and $g_{r}$ directly from the difference equation (1.06), as in the example given in $\$ 5$ below. This will settle immediately the question whether the above conditions can be fulfilled; then inspection of the sequence of trial values will generally indicate whether $n$ has been chosen large enough for $f_{r}$ and $g_{r}$ to have attained their asymptotic forms at $r=n$.

3. Error Analysis: Exact Arithmetic. The sequence $a_{r}$, constructed according to equations (1.08), can be expressed as a linear combination of the $f_{r}$ and $g_{r}$. 
Using the given values at $r=n$ and $n+1$, we find that

$$
a_{r}=\frac{g_{n+1} f_{r}-f_{n+1} g_{r}}{f_{n} g_{n+1}-f_{n+1} g_{n}}
$$

the non-vanishing of the denominator being a consequence of the assumed conditions of $\$ 2$ (compare (3.09) below). From (1.10) and (1.07), we find that

$$
\lambda=\left\{g_{n+1}\left(1-\sum_{j=n+1}^{\infty} m_{j} f_{j}\right)-f_{n+1} \sum_{j=0}^{n} m_{j} g_{j}\right\} /\left(f_{n} g_{n+1}-f_{n+1} g_{n}\right)
$$

The "error" in which we are interested is the difference between $\varphi_{r}$, that is, $a_{r} / \lambda$, and $f_{r}$. Rather than consider this directly, however, we shall seek bounds for the quantity $e_{r}$, defined by

$$
e_{r}=\lambda\left(f_{r}-\varphi_{r}\right)=\lambda f_{r}-a_{r}=a_{r}\left(f_{r}-\varphi_{r}\right) / \varphi_{r}
$$

An interpretation of $e_{r}$ is as follows. We have

$$
\log _{10}\left|e_{r}\right|=\log _{10}\left|a_{r}\right|-\log _{10}\left|\varphi_{r} /\left(f_{r}-\varphi_{r}\right)\right| \text {. }
$$

Hence $\log _{10}\left|e_{r}\right|$ is the excess of the number of digits in the integer part of $a_{r}$ over the number of "correct" significant figures in $\varphi_{r}$. In particular, if $\left|e_{r}\right| \leqq 1$ then the number of correct significant figures in $\varphi_{r}$ is not less than the number of digits in the integer part of the corresponding trial value $a_{r}$.

Using (3.01) and (3.02), we derive

$$
\begin{aligned}
e_{r}=\left\{f_{n+1} g_{r}-\left(\sum_{j=n+1}^{\infty} m_{j} f_{j}\right)\right. & g_{n+1} f_{r} \\
& \left.-\left(\sum_{j=0}^{n} m_{j} g_{j}\right) f_{n+1} f_{r}\right\} /\left(f_{n} g_{n+1}-f_{n+1} g_{n}\right) .
\end{aligned}
$$

Now when $0 \leqq r \leqq n$, we find from (2.01). and (2.02) that

$$
\left|f_{n+1} g_{r}\right| \leqq \rho \sigma^{n+1-r}\left|f_{n} g_{n+1}\right| \text {. }
$$

With the aid of (2.03), we obtain

$$
\left|\left(\sum_{j=n+1}^{\infty} m_{j} f_{j}\right) g_{n+1} f_{r}\right| \leqq a \tau\left|f_{n} g_{n+1}\right|
$$

where

$$
a=m \max _{0 \leqq r \leqq n}\left|f_{r}\right|
$$

Similarly,

$$
\left|\left(\sum_{j=0}^{n} m_{j} g_{j}\right) f_{n+1} f_{r}\right| \leqq\left(\sum_{j=0}^{n} m\left|g_{n+1}\right| \sigma^{n+1-j}\right) \rho\left|f_{n}\right| \frac{a}{m} \leqq \frac{a \rho \sigma}{1-\sigma}\left|f_{n} g_{n+1}\right|
$$

Next, we have $\left|f_{n+1} g_{n}\right| \leqq \rho \sigma\left|f_{n} g_{n+1}\right|$, and so

$$
\left|f_{n} g_{n+1}-f_{n+1} g_{n}\right| \geqq(1-\rho \sigma)\left|f_{n} g_{n+1}\right| \text {. }
$$

Substituting (3.05), (3.06), (3.08) and (3.09) in (3.04), we obtain our desired error bound:

$$
\left|e_{r}\right| \leqq(1-\rho \sigma)^{-1}\left[\left\{\sigma^{n-r}+(1-\sigma)^{-1} a\right\} \rho \sigma+a \tau\right] \quad(0 \leqq r \leqq n)
$$


With the assumed conditions of $\S 2$, the quantities $\rho, \sigma$ and $\tau$ are all small. Clearly if $a$ is not large compared with unity then $\left|e_{r}\right|$ will not exceed unity.

The interpretation of this extra condition depends on the relative magnitudes of the coefficients $m_{r}$. We may judge from examples, however, that it is a reasonable condition to impose on the use of the algorithm. Suppose, for example, we normalize by use of a prescribed value of $f_{0}$. Then we have $m=1 /\left|f_{0}\right|$, and

$$
a=\max _{0 \leqq r \leqq n}\left|f_{r} / f_{0}\right| \text {. }
$$

Thus $a$ could be large only if $f_{0}$ is small compared with other members of the sequence $f_{r}$, and we could not expect this method of normalization to be accurate in these circumstances. Alternatively, consider the case in which

$$
m_{0}=\frac{1}{2} m, \quad m_{j}= \pm m \quad(j \geqq 1) ;
$$

this occurs with (1.04), and also frequently with the boundary conditions in the Clenshaw method [13]. Then by requiring $a$ to be of order unity, we are stipulating that there must not be excessive cancellation in summing the series (1.07), and it is known [1] that if such cancellation occurs, then inaccuracies may result.

Summarizing this section so far, we have shown that with the conditions of $\$ 2$ and the restriction that the quantity $a$ defined by (3.07) must not be large compared with unity, $\left|e_{r}\right|$ cannot exceed unity. Accordingly, when the algorithm is carried out with exact arithmetic each $\varphi_{r}$ has at least as many correct significant figures as there are digits in the integer part of the corresponding $a_{r}$.

Remark. It might perhaps be thought that the condition that the second solution $g_{r}$ is increasing at $r=n$ is overly strong, and that $g_{r}$ could be permitted to decrease, provided that its rate of decrease were less than that of $f_{r}$. Certainly in these circumstances the algorithm may converge; see, for example, (1.11) and (1.12). In general, however, it would be untrue that $\left|e_{r}\right| \leqq 1$; this is readily seen by considering the contribution of the term

$$
\frac{f_{n+1} g_{r}}{f_{n} g_{n+1}-f_{n+1} g_{n}} \fallingdotseq \frac{f_{n+1}}{f_{n}} \frac{g_{r}}{g_{n+1}}
$$

on the right of (3.04). Thus in these circumstances conclusions concerning the accuracy of the sequence $\varphi_{r}$ would necessarily be weaker.

4. Effect of Rounding the Sequence of Trial Values. In applications, after computing each $a_{r}$ it is generally the practice to round its value to the nearest integer. To examine the effect of this upon the final sequence $\varphi_{r}$, it suffices to consider the propagation of a single rounding error, say $\epsilon_{s}$ in $a_{s}$, where $0 \leqq s \leqq n-1$, and $\left|\epsilon_{s}\right| \leqq \frac{1}{2}$.

In this analysis, we shall use the well-known identity

$$
f_{s} g_{s+1}-f_{s+1} g_{s}=q_{s+1}\left(f_{s+1} g_{s+2}-f_{s+2} g_{s+1}\right),
$$

obtained directly from the difference equation (1.06). Repeated application of this result gives

$$
f_{s} g_{s+1}-f_{s+1} g_{s}=q_{s+1} q_{s+2} \cdots q_{n}\left(f_{n} g_{n+1}-f_{n+1} g_{n}\right) .
$$

As a consequence of (3.09), we see that the cross-product on the left of this equation 
cannot vanish, provided that no coefficient $q_{r}$ vanishes; we shall assume this to be the case.

Equation (3.01) now holds only for $r>s$. For other values of $r$, we find that

$$
a_{r}=\frac{g_{n+1} f_{r}-f_{n+1} g_{r}}{f_{n} g_{n+1}-f_{n+1} g_{n}}+\frac{g_{s+1} f_{r}-f_{s+1} g_{r}}{f_{s} g_{s+1}-f_{s+1} g_{s}} \epsilon_{s} \quad(r \leqq s) .
$$

Accordingly, we obtain from (1.10) and (1.07)

$$
\begin{aligned}
\lambda=\frac{g_{n+1}\left(1-\sum_{j=n+1}^{\infty} m_{j} f_{j}\right)-f_{n+1} \sum_{j=0}^{n} m_{j} g_{j}}{f_{n} g_{n+1}-f_{n+1} g_{n}} & \\
& +\frac{g_{s+1}\left(1-\sum_{j=s+1}^{\infty} m_{j} f_{j}\right)-f_{s+1} \sum_{j=0}^{s} m_{j} g_{j}}{f_{s} g_{s+1}-f_{s+1} g_{s}} \epsilon_{s} .
\end{aligned}
$$

Again writing $e_{r}=\lambda f_{r}-a_{r}$, we find that

$$
e_{r}=e_{r}^{(0)}+\gamma_{r, 8} \epsilon_{s},
$$

where $e_{r}{ }^{(0)}$ denotes the right side of (3.04) (that is, the $e_{r}$ of $\$ 3$ ), and

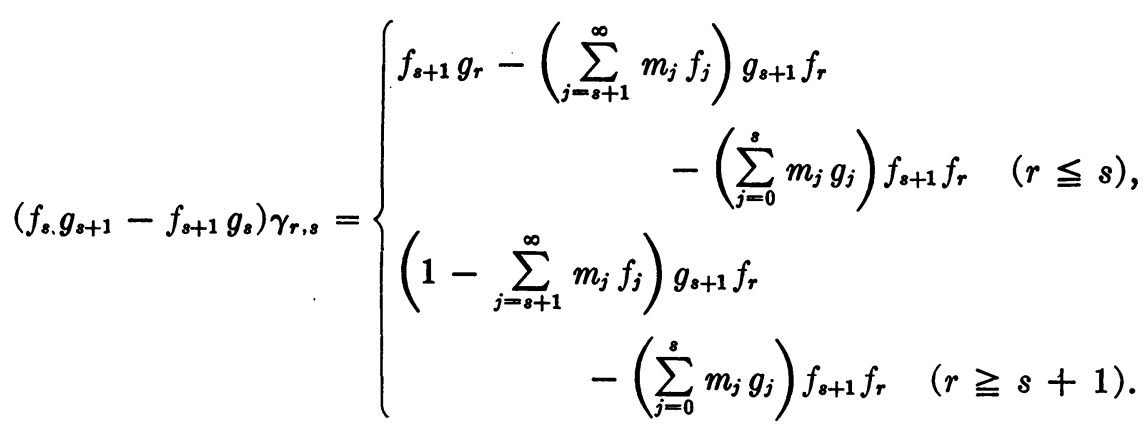

The bound (3.10) applies to $e_{r}{ }^{(0)}$. To derive corresponding bounds for $\gamma_{r, s}$, we denote by $v$ the largest quantity for which the inequalities

$$
\left|\frac{f_{r}}{f_{n}}\right| \leqq \frac{1}{v^{n-r}} \quad(r=0,1, \cdots, n)
$$

hold. Since we have assumed in $\$ 2$ that $\left|f_{r}\right|$ has acquired a moderately rapidly decreasing character by the time the point $r=n$ has been reached, it follows that $v$ is small.

From (2.02), (2.03), (3.07) and (4.07), we find that

$$
\begin{aligned}
& \left|f_{s+1} g_{r}\right| \leqq \sigma^{n+1-r} v^{s+1-n}\left|f_{n} g_{n+1}\right|, \quad\left|g_{s+1} f_{r}\right| \leqq \sigma^{n-s} v^{r-n}\left|f_{n} g_{n+1}\right| \\
& \left|\left(\sum_{j=s+1}^{\infty} m_{j} f_{j}\right) g_{s+1} f_{r}\right| \leqq\left(\sum_{j=s+1}^{n} m v^{j-n}+m \tau\right)\left|f_{n} g_{n+1}\right| \sigma^{n-s} \frac{a}{m} \\
& \leqq\left(\frac{1}{1-v}+\tau v^{n-s-1}\right) a \sigma^{n-s} v^{s+1-n}\left|f_{n} g_{n+1}\right| \text {, }
\end{aligned}
$$


and

$$
\begin{aligned}
\left|\left(\sum_{j=0}^{s} m_{j} g_{j}\right) f_{s+1} f_{r}\right| & \leqq\left(\sum_{j=0}^{s} m \sigma^{n+1-j}\left|g_{n+1}\right|\right) v^{s+1-n}\left|f_{n}\right| \frac{a}{m} \\
& \leqq \frac{a \sigma^{n+1-s} v^{s+1-n}}{1-\sigma}\left|f_{n} g_{n+1}\right| .
\end{aligned}
$$

Also, from (3.09) and (4.02) we have

$$
\left|f_{s} g_{s+1}-f_{8+1} g_{s}\right| \geqq\left|q_{s+1} q_{s+2} \cdots q_{n}\right|(1-\rho \sigma)\left|f_{n} g_{n+1}\right| .
$$

Substituting these inequalities in (4.06) and using the notation

$$
b=\max _{0 \leqq s \leqq n-1}\left\{\frac{1}{\left|q_{8+1} q_{8+2} \cdots q_{n}\right|}\left(\frac{\sigma}{v}\right)^{n-8}\right\},
$$

we find that

$$
\left|\gamma_{r, 8}\right| \leqq b v(1-\rho \sigma)^{-1}\left[\sigma^{8-r+1}+a\left\{\tau v^{n-s-1}+(1-v)^{-1}+\sigma(1-\sigma)^{-1}\right\}\right]
$$

and

$$
\begin{array}{r}
\left|\gamma_{r, 8}\right| \leqq b v(1-\rho \sigma)^{-1}\left[v^{r-8-1}+a\left\{\tau v^{n-s-1}+(1-v)^{-1}+\sigma(1-\sigma)^{-1}\right\}\right] \\
(r \geqq s+1) .
\end{array}
$$

In $\S 3$ we observed that if $\rho, \sigma, \tau$ are small and $a$ is of order unity, then $\left|e_{r}{ }^{(0)}\right|<1$. Inspection of (4.13) and (4.14) shows that since $v$ is small we shall also have $\left|\gamma_{r, s}\right|<1$, provided that $b$ is not large compared with unity. In order to evaluate this additional condition, consider the case in which $p_{r}$ and $q_{r}$ are both independent of $r$. The difference equation (1.06) is then solvable explicitly, and we find that $\sigma / v=q_{r}$ and $b=1$. In the general case we have to assume that $p_{r}$ and $q_{r}$ are relatively slowly-varying functions of $r$. Then $f_{r}$ and $g_{r}$ behave locally as solutions of a difference equation with constant coefficients and hence we may expect $b$ to be of order unity.

Thus we conclude that if the sequence of trial values $a_{r}$ is rounded to the nearest integer at each step of the algorithm, then each $\varphi_{r}$ still has at least as many correct significant figures as there are digits in the $a_{r} \dagger$. In other words, the rounding procedure is justified, in the sense that it does not adversely affect our "guaranteed" accuracy of the algorithm. This point is discussed further in the next section.

5. Example. We consider the solution of the difference equation

$$
(2 r-1) f_{r-1}-12 r f_{r}+(2 r+1) f_{r+1}=0,
$$

with the condition

$$
\frac{1}{2} f_{0}+f_{1}+f_{2}+f_{3}+\cdots=1 .
$$

This problem arose in the solution of a first-order differential equation by Clenshaw's method ([14], §15).

$\dagger$ In the case of the modified Bessel functions, this result is stated, without proof, in [3] as a consequence of practical observation. 
We apply formulas (1.08) to (1.10), taking

$$
p_{r}=-12 r /(2 r-1), \quad q_{r}=(2 r+1) /(2 r-1)
$$

and $n=4$. The following sequences of trial values are then obtained.

\begin{tabular}{cccc}
\hline$r$ & $a_{r}($ exact $)$ & $a_{r}$ (rounded) & $\epsilon_{r}$ \\
\hline 0 & $4324 \frac{1}{5}$ & 4413 & 0 \\
1 & $372 \frac{1}{3}$ & 380 & $-\frac{1}{3}$ \\
2 & $47 \frac{34}{5}$ & 49 & 0 \\
3 & $6 \frac{6}{7}$ & 7 & $\frac{1}{7}$ \\
4 & 1 & 1 & 0 \\
5 & 0 & 0 & 0 \\
\hline
\end{tabular}

Exact arithmetic has been used to compute the column headed $a_{r}$ (exact). In forming the next column, however, the $a_{r}$ have been rounded to the nearest integer at each step, the corresponding rounding errors $\epsilon_{r}$ being given in the fourth column.

We now form $\lambda$ by means of (1.10), taking $m_{0}=\frac{1}{2}$ and $m_{j}=1 \quad(j \geqq 1)$. This gives

$$
\left.\left.\lambda=2590 \frac{19}{7} \quad \text { (for exact } a_{r}\right), \quad \lambda=2643 \frac{1}{2} \quad \text { (for rounded } a_{r}\right) .
$$

The algorithm is completed by computing $\varphi_{r}=a_{r} / \lambda$, and the results are given in

\begin{tabular}{|c|c|c|c|c|c|}
\hline \multirow[b]{2}{*}{$r$} & & \multicolumn{2}{|c|}{-exact $a_{r}$} & \multicolumn{2}{|c|}{$\left\ulcorner^{\text {rounded } a_{r}}\right.$} \\
\hline & $f_{r}$ & $\varphi_{r}$ & $e_{r}$ & $\varphi_{r}$ & $e_{r}$ \\
\hline 0 & 1.6692537 & 1.6694003 & -0.380 & 1.6693777 & -0.328 \\
\hline 1 & 0.1437342 & 0.1437467 & -0.032 & 0.1437488 & -0.039 \\
\hline 2 & 0.0185187 & 0.0185198 & -0.003 & 0.0185360 & -0.046 \\
\hline 3 & 0.0026494 & 0.0026473 & 0.005 & 0.0026480 & 0.004 \\
\hline & 0.0003979 & 0.0003861 & 0.031 & 0.0003783 & 0.052 \\
\hline
\end{tabular}
the third and fifth columns in the next table.

For comparison, we also give in this table accurate seven-decimal values of the $f_{r}$, obtained by use of the algorithm with a higher value of $n$. Thence we are able to evaluate our measure $e_{r}$, defined by (3.03), for the discrepancy between $f_{r}$ and $\varphi_{r}$.

Our principal observation concerning these numerical results is that $\left|e_{r}\right|$ nowhere exceeds unity. This result is predicted by the analysis of the preceding sections, because the assumed conditions are readily seen to be satisfied in this example. For large $r$ the difference equation (5.01) becomes asymptotically

$$
f_{r-1}-6 f_{r}+f_{r+1}=0,
$$

with solutions

$f_{r}=($ constant $) \times \alpha^{-r}, \quad g_{r}=$ (constant) $\times \alpha^{r}, \quad$ where $\alpha=3+2 \sqrt{2}=5.83 \cdots$.

It is clear from the computed numerical values that this asymptotic character is attained by the decreasing solution $f_{r}$ by the stage $r=4$. It is also quite easy to see, without carrying out detailed arithmetic, that the solution $g_{r}$ will have attained its rapidly increasing character by this stage. Therefore the quantities 
$\rho, \sigma, \tau$ and $v$ defined in $\S \S 2$ and 4 are all small. The other conditions, that $a$ and $b$ (defined in $\S \S 3$ and 4 ) be of order unity, are seen to be fulfilled, first because there was no concellation in summing the series (1.10), and secondly because the coefficients $p_{r}$ and $q_{r}$ are relatively slowly-varying functions of $r$.

As a further illustration, let us normalize by means of the given value $f_{0}=1.6692537$, instead of by use of $(5.02)$. We then obtain the following results.

\begin{tabular}{ccccccc} 
& \multicolumn{2}{c}{ exact $a_{r}$} & \multicolumn{2}{c}{$e_{r}$} & $\varphi_{r}$ & $e_{r}$ \\
$r$ & $\varphi_{r}$ & $e_{r}$ & 1.6692537 & 0 \\
0 & 1.6692537 & 0 & 0.1437381 & -0.010 \\
1 & 0.1437340 & 0.001 & 0.0185347 & -0.042 \\
2 & 0.0185182 & 0.001 & 0.0026478 & 0.004 \\
3 & 0.0026470 & 0.006 & 0.0003783 & 0.052
\end{tabular}

Again $\left|e_{r}\right|<1$, as expected. It may also be observed that this method of normalization yields somewhat smaller errors $\left|e_{r}\right|$ than the previous method in the case when the $a_{r}$ are computed exactly. The bound (3.10) indicates that this is generally true, unless the quantity $a$ happens to be unduly large, because of the vanishing of the term in $\tau$. On the other hand, we may expect the contributions of the rounding errors $\epsilon_{s}$ in the two methods to be comparable, because the dominant terms $a(1-v)^{-1}$ and $v^{r-s-1}$ (when $r=s+1$ ) in the square brackets in (4.13) and (4.14) are of comparable size in the two cases. By forming the differences between the values of $e_{r}$ obtained from the exact and rounded $a_{r}$ in the two methods, we see that this conclusion, also, is borne out by the example.

As a final observation, we remark that the column of values given in this section headed $a_{r}$ (exact) differs from the corresponding numerical values given by Fox in the column $a_{r}{ }^{(0)}$ in Table 2 of [14]. This is because Fox, in effect, uses different initial conditions for his sequence. By superimposing an artificial "rounding error" $\epsilon_{2}=-9 / 5$ on our values of $a_{r}$ (exact), however, we reproduce Fox's sequence (apart from differences in sign); in this way his results are brought within the scope of the present error analysis.

6. Conclusion. The Miller algorithm for the computation of the solution of the difference equation (1.06) which satisfies the condition (1.07) is given by (1.08), (1.09) and (1.10). We have shown that if the wanted solution $f_{r}$ and an independent solution $g_{r}$ satisfy the relations (2.01) and (2.02), in which $\rho$ and $\sigma$ are positive numbers less than unity, then the errors in the values $\varphi_{r}$ yielded by the algorithm are bounded by the strict inequality (3.10), in which $e_{r}=\lambda\left(f_{r}-\varphi_{r}\right)$ and $\tau$ and $a$ are defined by (2.03) and (3.07).

We have also investigated the effects of rounding the sequence of trial values $a_{r}$, and have found that an error $\epsilon_{s}$ introduced in $a_{s}$ increases $e_{r}$ by $\gamma_{r, s} \epsilon_{s}$, where $\gamma_{r, s}$ is subject to the strict inequalities (4.13) and (4.14) in which $v(<1)$ and $b$ are defined by (4.07) and (4.12), respectively.

We have seen that in circumstances in which the algorithm is commonly applied, $\rho, \sigma, \tau$ and $v$ are small, and $a$ and $b$ are not large compared with unity. In consequence $\left|e_{r}\right|<1$ and $\left|\gamma_{r, s}\right|<1$, which mean that the number of correct significant figures 
in each $\varphi_{r}$ yielded by the algorithm is not less than the number of digits in the integer part of the corresponding trial value $a_{r}$, whether or not the $a_{r}$ are rounded during the computation. Thus the practice of repeating the algorithm with different starting positions $n$ merely to ascertain the accuracy of the $\varphi_{r}$ is often quite unnecessary.

This theory has application to the automatic computation of many transcendental functions, and also to Clenshaw's method of solving ordinary linear differential equations in series of Chebyshev polynomials in the case when the coefficients in this series satisfy a three-term recurrence relation. A comprehensive error analysis of the Clenshaw method has not been attempted, but by making assumptions of the type used in this paper it is quite possible that similar analysis will yield useful bounds in more complicated cases.

Acknowledgement. The author thanks Mr. C. W. Clenshaw and Mr. G. F. Miller of the National Physical Laboratory, Teddington, for reading the manuscript of this paper and making valuable criticisms.

National Bureau of Standards

Washington, D. C.

1. British Association For The Advancement Of Science, "Bessel functions, Part II," Mathematical Tables, v. X, Cambridge University Press, 1952.

2. C. W. Jones, "A short table for the Bessel functions $I_{n+\xi}(x),(2 / \pi) K_{n+\xi}(x)$," Royal Society Shorter Mathematical Tables, No. 1, Cambridge University Press, 1952.

3. L. Fox, "A short table for Bessel functions of integer orders and large arguments," Royal Society Shorter Mathematical Tables, No. 3, Cambridge University Press, 1954.

4. F. J. Corbat6, "On the computation of auxiliary functions for two-center integrals by means of a high-speed computer," J. Chem. Phys., v. 24, 1956, p. 452-453.

5. I. A. Stegun \& M. Abramowitz, "Generation of Bessel functions on high speed computers,"; $M T A C$, v. 11, 1957, p. 255-257.

6. J. B. Randels \& R. F. Reeves, "Note on empirical bounds for generating Bessel functions," Comm. Assoc. Comput. Mach., v. 1, May 1958, p. 3-5.

7. M. Goldstein \& R. M. Thaler, "Recurrence techniques for the calculation of Bessel functions," $M T A C$, v. 13, 1959, p. 102-108.

8. F. J. Corbat6 \& J. L. URetsky, "Generátion of spherical Bessel functions in digital computers," J. Assoc. Comput. Mach., v. 6, 1959, p. 366-375.

9. National Bureau Of Standards, "Handbook of mathematical functions," Appl. Math. Ser. 55, Government Printing Office, Washington, D. C. (In press.) (Especially Section 9.12, Examples 1 and 7.)

10. M. Abramowitz, Review of a paper by J. Kaye, $M T A C$, v. 10, 1956, p. 176-177.

11. W. GAUTSCHI, "Recursive computation of the repeated integrals of the error function," Math. Comp., v. 15, 1961, p. 227-232.

12. A. Rotenberg, "The calculation of toroidal harmonics," Math. Comp. v. 14, 1960, p. 274-276.

13. C. W. Clenshaw, "The numerical solution of linear differential equations in Chebyshev series," Proc. Cambridge Phil. Soc., v. 53, 1957, p. 134-149.

14. L. Fox, "Chebyshev methods for ordinary differential equations," Computer J., v. 4, 1962 , p. 318-331. 